\title{
PRELIMINARY LIST OF TERMS FOR THE ARC SPECTRUM OF TANTALUM
}

\author{
By C. C. Kiess and Harriet K. Kiess
}

\section{ABSTRACT}

A preliminary list of Ta I terms, accounting for most of the strong arc lines, is presented. The low ${ }^{4} \mathrm{~F}$ and ${ }^{4} \mathrm{P}$ terms and the metastable ${ }^{6} \mathrm{D}$ and ${ }^{4} \mathrm{D}$ terms are identified with those to be expected theoretically from the electron configurations $5 d^{3} \cdot 6 s^{2}$ and $5 d^{4} \cdot 6 s$.

\section{CONTENTS}

II. Term table for Ta I

\section{INTRODUCTORY NOTE}

The arc and spark spectra of tantalum have been observed at the Bureau of Standards from 2,000 A in the ultraviolet to 10,300 A in the infrared. These spectra are exceedingly rich in lines and the work of compiling the wave lengths and separating them according to the degree of ionization of the emitting atoms is still incomplete. Many of the arc lines are clearly complex, but are not sufficiently resolved on our plates to give their exact structure. Zeeman effects for many of the tantalum lines have been observed also, but only a few of them, apparently, are based on the $g$-values of simple (LS) coupling.

With the aid of these new data, we have been able to classify most of the strong Ta I lines, including those that are complex. The terms upon which this classification is based are given in the table below and are offered at this time in response to several requests that have been made of the Bureau of Standards for them. Theoretically the terms result from the interaction of five valence electrons which occupy the $\mathrm{O}$ and $\mathrm{P}$ shells. In the unexcited state of the atom the electrons form the configuration $5 d^{3} \cdot 6 s^{2}$ from which arise the even terms ${ }^{4} \mathrm{~F},{ }^{4} \mathrm{P},{ }^{2} \mathrm{H},{ }^{2} \mathrm{G}$, etc. The configuration $5 d^{4} \cdot 6 s$ gives the metastable terms ${ }^{6} \mathrm{D},{ }^{4} \mathrm{D},{ }^{4} \mathrm{H},{ }^{4} \mathrm{G},{ }^{2} \mathrm{I}$, etc. The more prominent terms of these groups have now been found and it is their combinations with higher odd terms that account for most of the strong $\mathrm{Ta}$ I lines. At this stage of the analysis, it is not possible to arrange with certainty these odd terms into multiplet groups and to assign specifically to each its electronic origin. Accordingly, in this preliminary note, the odd terms are designated only by their inner quantum numbers, affixed to the letters Z, Y, X, etc., except in a few instances where the term character is clearly indicated by the Zeeman effect. The numerical values of the terms are arbitrarily based on the assumption that the lowest term is ${ }^{4} \mathrm{~F}_{11 / 2}=0.00$. 


\section{TERM TABLE FOR Ta I}

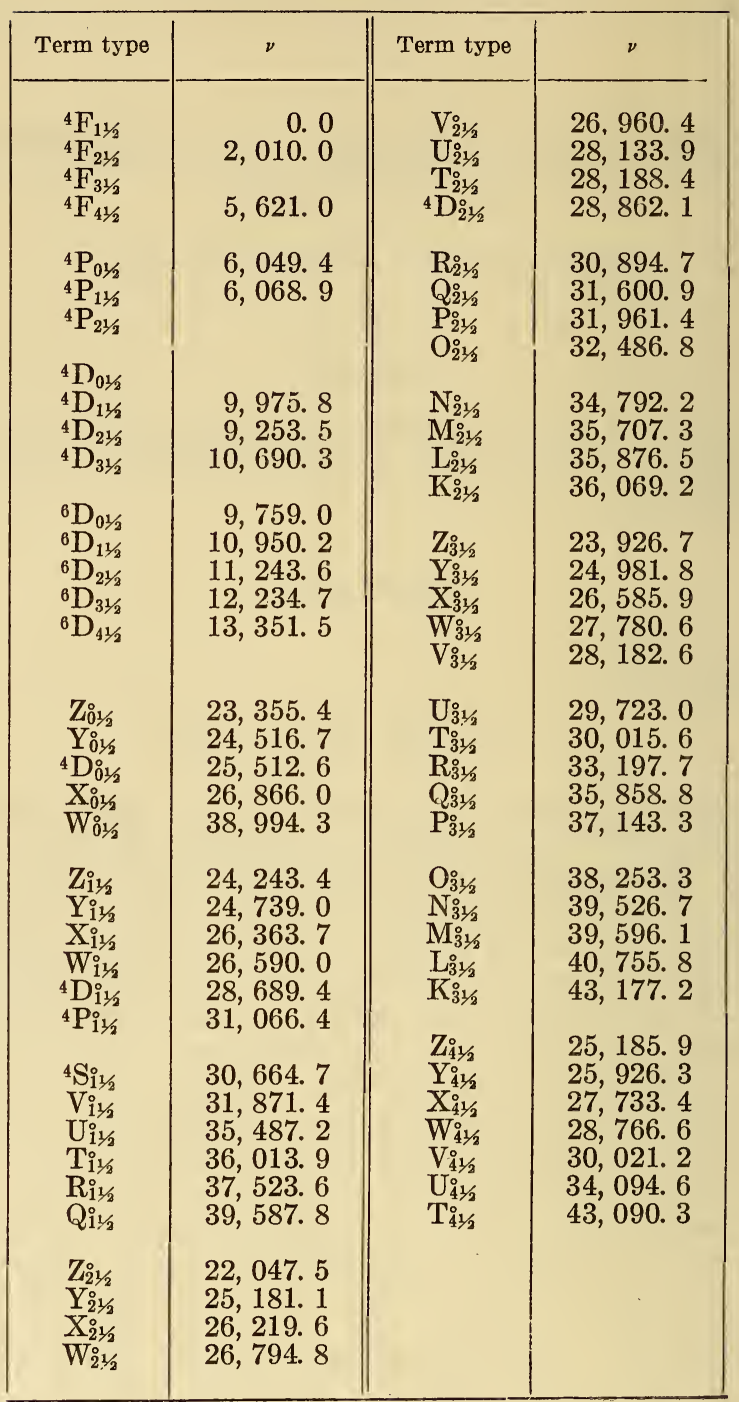

Washington, June 9, 1933. 